\title{
Detection of a Single Texture in Colour Images
}

\author{
Linjiang Yu and Georgy Gimel'farb \\ CITR, Department of Computer Science \\ Tamaki Campus, The University of Auckland \\ Auckland, New Zealand \\ lyu011@ec.auckland.ac.nz, g.gimelfarb@auckland.ac.nz
}

\begin{abstract}
Detection of regions similar to a single given texture in arbitrary colour images is difficult for conventional supervised or unsupervised segmentation techniques. We introduce a novel partially supervised algorithm that solves this problem using similarity between local statistics on different levels of pyramidal representations of the texture and the image. Most characteristic statistics for the texture are estimated in accord with a generic Gibbs random field model with spatially homogeneous pairwise pixel interactions. Empirical distributions of the self-similarity values for the texture itself are used to separate the desired texture from an arbitrary background. Experiments with different images, including aerial images of the Earth's surface, show this algorithm effectively detects regions with spatially homogeneous and weakly homogeneous textures.
\end{abstract}

\section{Introduction}

Both unsupervised and supervised texture segmentation has been intensively studied for long time. In both the cases the goal is to separate an image into regions containing each an individual spatially homogeneous texture. But most typical practical segmentation problems belong to partially supervised segmentation such that the training information is available only for few regions of interest, while the information about all other non-target regions is absent. The partially supervised segmentation is necessary for identifying regions of interest in remote sensing of the Earth's surface, medical diagnostics, and industrial vision. One more typical application is the content-based image retrieval (CBIR) (see, e.g., comprehensive surveys in $[1,9,10]$ ). The partially supervised segmentation allows for retrieving images from a large image database that contain regions similar to a given small query image [13].

Up to now, partially supervised segmentation has not been under intensive studies although it cannot be performed with existing supervised or unsupervised techniques. Nonetheless, several schemes for one-class classification were proposed for some applications $[2,6,11]$.

The algorithm in [14] separates homogeneous texture from an arbitrary background using local and global distributions of colours and colour cooccurrences. In line with other statistical approaches, it is suitable for translation invariant 
textures. But many of real visually homogeneous textures are weakly variant with respect to translation, and their spatial homogeneity is recovered better using a pyramidal image representation. This paper attempts to detect a weakly inhomogeneous texture in an arbitrary colour image as well as compare a few different measures of similarity between two probability distributions.

\section{Basic Steps of Detection Algorithm}

The basic steps are as follows given a value of $\bar{\zeta}$ :

1. Create a codebook with a fixed number of codevectors for the training sample using colour space vector quantization (CSVQ).

2. Convert the training sample into the index image with respect to the codebook.

3. Find the maximum deviation of each codevector with respect to the training sample.

4. Create the index image for the original image with the codevectors and their deviations.

5. The above two index images become the base levels of pyramids. Find the number $K$ of pyramid levels based on the sizes of the training area and a fixed moving window.

6. Find a characteristic subset of colour cooccurrence histograms (CCHs) for the base training level image of the index training sample and collect the corresponding global normalized $\mathrm{CCHs}$ (nCCHs). Set $k=0$.

7. Find a cumulative empirical distribution of distances between the local $\mathrm{nC}$ $\mathrm{CHs}$ over the moving window around each pixel and the like global nCCHs over the whole training image at level $k$.

8. Calculate the distances between the local $\mathrm{nCCHs}$ for the candidate regions and the global nCCHs for the training image at level $k$. Get the cumulative distance map.

9. Calculate the rejection rate $\rho$ if $k=0$, then select a distance threshold $\zeta_{k}$ using the distribution at step 7 .

10. If $\zeta_{k} \leq \bar{\zeta}$ or $k \geq K$, go to next step, otherwise go to step 13 .

11. Set the next level: $k=k+1$.

12. Build up the index image of the candidate regions and training image at level $k$. Go to step 7.

13. Detect the regions by thresholding the cumulative distance map at step 8 with $\zeta_{k}$.

Details of the CSVQ (step 1) has been already discussed in paper [14]. The codebook $\mathbf{B}=\left[\mathbf{b}_{k}: k=1, \ldots, N\right]$ was obtained in [14] by approximating an empirical colour distribution for the training sample $\mathbf{S}^{t r}$ with a mixture of Gaussians (GM). But such an approximation is not effective in the case of a small number of sparsely or uniformly distributed codevectors. To avoid the above difficulties, the colour thresholding in this paper uses the codebook $\mathbf{B}$ and its deviation array $\Delta=\left[\delta_{k}: k=1, \ldots, N\right]$ with respect to the training sample. Each element $\delta_{k}$ is 
the maximum deviation of the codevector $\mathbf{b}_{k}$ within its corresponding partition in the training sample.

In the index image, each initial colour is replaced by the index of the closest codebook colour (in terms of the Cartesian distance) if the distance is less than a predefined threshold, $\delta$, or is considered as background otherwise.

Let $\mathbf{G}(0)=\left[g_{i}(0): i \in \mathbf{R}_{0} ; g_{i}(0) \in \mathbf{N}\right]$ and $\mathbf{G}^{\operatorname{tr}}(0)=\left[g_{j}^{\text {tr }}(0): j \in\right.$ $\left.\mathbf{R}_{0}^{t r} ; g_{j}^{t r}(0) \in \mathbf{N}\right]$ be the base index image for the original colour image to be segmented and the base training image, respectively, on the supporting finite arithmetic lattices $\mathbf{R}_{0}=\left\{(x, y): x=0, \ldots, X_{0}-1 ; y=0, \ldots, Y_{0}-1\right\}$ and $\mathbf{R}_{0}^{t r}=\left\{(x, y): x=0, \ldots, X_{0}^{t r}-1 ; y=0, \ldots, Y_{0}^{t r}-1\right\}$. At level $k+1$ of the pyramid, the image $\mathbf{G}(k+1)=\left[g_{x^{\prime}, y^{\prime}}(k+1):\left(x^{\prime}, y^{\prime}\right) \in \mathbf{R}_{k+1} ; g_{x^{\prime}, y^{\prime}}(k+1) \in \mathbf{N}\right]$ can be obtained from the preceding image $\mathbf{G}(k)=\left[g_{x, y}(k):(x, y) \in \mathbf{R}_{k} ; g_{x, y}(k) \in \mathbf{N}\right]$ at level $k$ by downsampling: $X_{k+1}=\left\lfloor X_{k} / 2\right\rfloor, Y_{k+1}=\left\lfloor Y_{k} / 2\right\rfloor$ and $g_{x^{\prime}, y^{\prime}}(k+1)=$ $g_{\lfloor x / 2\rfloor,\lfloor y / 2\rfloor}(k)$ for all $\left(x^{\prime}, y^{\prime}\right)$.

\section{Texture Similarity Measure}

Let $\mathbf{A}=\left\{\left(\xi_{a}, \eta_{a}\right): a=1, \ldots, A\right\}$ be the most "energetic" translation invariant families of interacting pixel pairs (see [14] in detail). Let $\mathbf{F}_{a}(\mathbf{G})=\left[F_{a}(q, s \mid \mathbf{G})\right.$ : $q, s \in \mathbf{N}]$ and $\mathbf{F}_{a, i}(\mathbf{G})=\left[F_{a, i}(q, s \mid \mathbf{G}): q, s \in \mathbf{N}\right]$ denote the global normalized colour cooccurrence histogram $(\mathrm{nCCH})$ for the family $\mathbf{C}_{a}$ and the local $\mathrm{nCCH}$ over the rectangular moving window $\widetilde{\mathbf{W}}=W_{x} \times W_{y}$ around a position $i$ in the image $\mathbf{G}$, respectively. The interaction energy $\varepsilon_{a}(\mathbf{G})$ for the family $\mathbf{C}_{a}$ (see $[3]$ in detail) is defined as:

$$
\varepsilon_{a}(\mathbf{G})=\sum_{(q, s) \in \mathbf{N}^{2}} F_{a}(q, s \mid \mathbf{G})\left(F_{a}(q, s \mid \mathbf{G})-\frac{1}{|\mathbf{N}|^{2}}\right)
$$

The number $K$ of pyramid levels depends on the radius $r$ of the base training area and the width of the moving window $\widetilde{\mathbf{W}}$ so that the training pixels at the highest pyramidal level are still sufficient for collecting the CCHs to represent the texture at this level:

$$
K=\frac{\ln (2 r)-\ln \left(\min \left(W_{x}, W_{y}\right)\right)}{\ln 2}
$$

Let the characteristic subset of pixel neighbours $\mathbf{A}(0)=\left\{\left(\xi_{a}^{0}, \eta_{a}^{0}\right): a=\right.$ $1, \ldots, A\}$ be the clique families with the top-rank interaction energies $\varepsilon_{a}\left(\mathbf{G}^{\operatorname{tr}}(0)\right)$ over the index training image $\mathbf{G}^{\operatorname{tr}}(0)$ and the maximum energy $\varepsilon^{\max }=\max \left\{\varepsilon_{a}\left(\mathbf{G}^{\operatorname{tr}}(0)\right): a=1, \ldots, A\right\}$. The corresponding subset $\mathbf{A}(k)$ at level $k$ is denoted as $\mathbf{A}(k)=\left\{\left(\xi_{a}^{k}, \eta_{a}^{k}\right): a=1, \ldots, A\right\}$ so that

$\xi_{a}^{k}=\xi_{a}^{0}$, if $\left\lfloor\xi_{a}^{0} / 2^{k}\right\rfloor<2 ; \xi_{a}^{k}=\left\lfloor\xi_{a}^{0} / 2^{k}\right\rfloor$, otherwise.
$\eta_{a}^{k}=\eta_{a}^{0}$, if $\left\lfloor\eta_{a}^{0} / 2^{k}\right\rfloor<2 ; \eta_{a}^{k}=\left\lfloor\eta_{a}^{0} / 2^{k}\right\rfloor$, otherwise.

The paper [12] has investigated the changes of pairwise pixelwise interaction structures along the Gaussian pyramid. The structure $\mathbf{A}(k)$ at level $k$ reflects 
more long-range interaction than $\mathbf{A}(k-1)$ at level $k-1$. Pyramidal similarity may be justified only if textures become more homogeneous when adding more up levels so that the assumption of translation invariant pixel interactions can be satisfied. For weakly homogeneous or inhomogeneous textures, the most characteristic interactions belong to the long-range ones which cannot be captured in the base level given a small moving window but can be reduced and captured at the up levels, so the detection performance may be improved. However, for homogeneous textures, their short-range ones are already captured at the base level, adding more up levels may not improve the performance and may decrease the discriminability due to the lack of their characteristic short-range interactions at up levels. Therefore, this algorithm will start from the base level of pyramid and go up level by level until a criterion is satisfied. Let the pixelwise distance measure be the cumulative distance along the pyramid levels $k=0, \ldots, L$ :

$$
\begin{aligned}
D_{i}^{L}\left(\mathbf{F}_{i}(\mathbf{G}), \mathbf{F}\left(\mathbf{G}^{t r}\right)\right)= & \sum_{k=0}^{L} \frac{1}{|\mathbf{A}(k)|} \sum_{a \in \mathbf{A}(k)} \frac{\varepsilon^{\max }}{\varepsilon_{a}\left(\mathbf{G}^{t r}(k)\right)} \bullet \\
& D_{a, \frac{i}{2^{k}}}\left(\mathbf{F}_{a, \frac{i}{2^{k}}}(\mathbf{G}(k)), \mathbf{F}_{a}\left(\mathbf{G}^{t r}(k)\right)\right)
\end{aligned}
$$

where $D_{a, \frac{i}{2^{k}}}\left(\mathbf{F}_{a, \frac{i}{2^{k}}}(\mathbf{G}(k)), \mathbf{F}_{a}\left(\mathbf{G}^{t r}(k)\right)\right)$ is the distance between the local nCCH at the position $\frac{i}{2^{k}}$ over the image $\mathbf{G}(k)$ at level $k$ and the global nCCH over the training image $\mathbf{G}^{t r}(k)$ for the clique family $\mathbf{C}_{a}, i \in \mathbf{R}_{0}$, and it is scaled with the ratio $\frac{\varepsilon_{\text {max }}}{\varepsilon_{a}\left(\mathbf{G}^{t r}(k)\right)}$ to be comparable each other.

Let $\mathbf{f}_{a}=\left\{f_{a, i}: i=1, \ldots, n ; \sum_{i=1}^{n} f_{a, i}=1\right\}$ and $\mathbf{f}_{b}=\left\{f_{b, i}: i=1, \ldots, n\right.$; $\left.\sum_{i=1}^{n} f_{b, i}=1\right\}$ be two discrete density functions. The dissimilarity measure $d\left(\mathbf{f}_{a}, \mathbf{f}_{b}\right)$ between the two functions can be done in many ways. The experiments in [7] had shown that the fidelity measure tends to give sharper results if two density functions to be compared are quite close each other, comparing to $\chi^{2}[8]$ and Jensen-Shannon divergence (JS) [5] measures. The quadratic form distance (QF) [4] can be the best solution because its similarity matrix may consider the closeness between the training colours.

(1) The symmetric $\chi^{2}$-distance:

$$
d\left(\mathbf{f}_{a}, \mathbf{f}_{b}\right)=\sum_{i=1}^{n} \frac{\left(f_{a, i}-f_{b, i}\right)^{2}}{f_{a, i}+f_{b, i}}, 0 \leq d\left(\mathbf{f}_{a}, \mathbf{f}_{b}\right) \leq 2
$$

(2) The fidelity measure:

$$
d\left(\mathbf{f}_{a}, \mathbf{f}_{b}\right)=\sqrt{1-\left(\sum_{i=1}^{n} \sqrt{f_{a, i}} \sqrt{f_{b, i}}\right)^{2}}, 0 \leq d\left(\mathbf{f}_{a}, \mathbf{f}_{b}\right) \leq 1
$$

(3) The quadratic form distance (QF):

$$
d\left(\mathbf{f}_{a}, \mathbf{f}_{b}\right)=\sqrt{\left(\mathbf{f}_{a}-\mathbf{f}_{b}\right)^{T} \mathbf{A}\left(\mathbf{f}_{a}-\mathbf{f}_{b}\right)}, 0 \leq d\left(\mathbf{f}_{a}, \mathbf{f}_{b}\right) \leq \sqrt{2}
$$




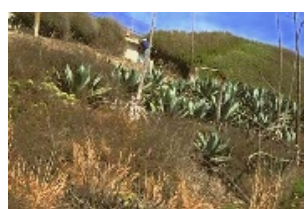

(a) GrassPlantsSky.1

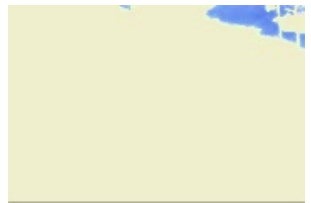

(d) For "sky": $K=1$, $|\mathbf{N}|=14, \zeta_{0}^{12 \%}=1.13$

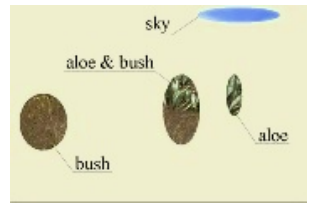

(b) Training samples

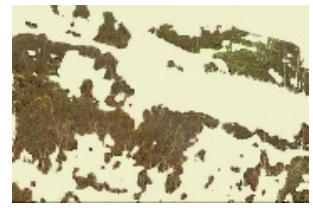

(e) For "bush": $K=3$, $|\mathbf{N}|=16, \zeta_{0}^{0.4 \%}=0.47$

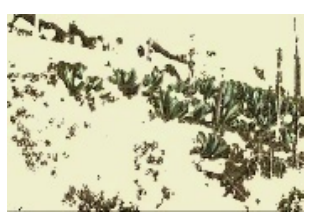

(c) For "aloe": $K=1$, $|\mathbf{N}|=16, \zeta_{0}^{1.3 \%}=0.55$

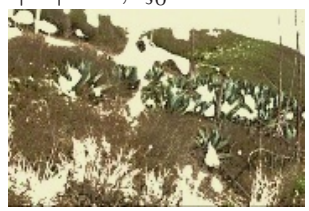

(f) For "aloe \& bush": $K=2,|\mathbf{N}|=16, \zeta_{1}^{4.4 \%}=0.50$

Fig. 1. Results of detecting for different training samples using $\chi^{2}$-distance measure from the image GrassPlantsSky.1.

where $\mathbf{A}=\left[a_{i j}\right]$ is the similarity matrix, and $a_{i j}$ denote the cross-bin similarity between bins $i$ and $j$. Let $d_{i j}$ be the Euclidean distance between colour $i$ (or a pair of colours $i$ ) and colour $j$ (or a pair of colours $j$ ), $d_{\max }=\max _{i, j}\left(d_{i j}\right)$, then $a_{i j}=1-\frac{d_{i j}}{d_{\max }}$.

The resulting regions can be obtained from the distance map $\left\{D_{i}^{L}\left(\mathbf{F}_{i}(\mathbf{G}), \mathbf{F}\left(\mathbf{G}^{t r}\right)\right): i \in \mathbf{R}_{0}\right\}$ by a distance threshold $\zeta_{L}^{\rho}$. All the pixels where their distances are less than $\zeta_{L}^{\rho}$ will be remained. Let $\zeta_{L}^{\rho}$ be chosen from the empirical distribution of distances $\left\{D_{j}^{L}\left(\mathbf{F}_{j}\left(\mathbf{G}^{t r}\right), \mathbf{F}\left(\mathbf{G}^{t r}\right)\right), j \in \mathbf{R}_{0}^{t r}\right\}$ over the training texture pyramid $\left\{\mathbf{G}^{t r}(0), \ldots, \mathbf{G}^{t r}(L)\right\}$ by rejecting a certain small percentage $\rho$ of the topmost training distances. The rejection rate $\rho$ depends on the homogeneity of the training sample. Let $D_{\max }^{0}=\max \left\{D_{j}^{0}\left(\mathbf{F}_{j}\left(\mathbf{G}^{t r}\right), \mathbf{F}\left(\mathbf{G}^{t r}\right)\right)\right.$ : $\left.j \in \mathbf{R}_{0}^{t r}\right\}$ be the maximum distance over the base level of the training sample. Assume that a fixed value $\bar{\zeta}$ is the threshold of homogeneity depending on specific distance measure and $\hat{D}$ is the upbound of a distance measure, and $\hat{\rho}$ is the pre-defined maximum rejection rate. Then the rejection rate $\rho$ is defined as:

1. $\rho=0 \%$, if $D_{\max }^{0} \leq \bar{\zeta}$ (homogeneous training sample);

2. $\rho=\frac{\hat{\rho}\left(D_{\max }^{0}-\bar{\zeta}\right)}{\hat{D}-\bar{\zeta}} \times 100 \%$, otherwise (inhomogeneous training sample, e.g. $\hat{\rho}=$ $20 \%)$.

\section{Experimental Results and Conclusions}

The experimental analysis and comparisons are based on visual observation due to the lack of the "ground truth" for the specific detection problems. Furthermore, the detecting requirements usually depend on specific applications.

The first examples use two colour images $(768 \times 512)$ GrassPlantsSky.1 (a) in Fig. 1 and ValleyWater.2 (a) in Fig. 2 from the MIT Media Lab VisTex database, and (b) shows the different training samples cut from the original ones. The 


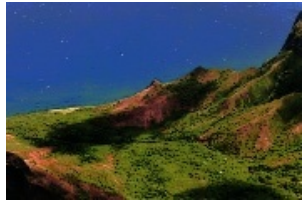

(a) ValleyWater.2

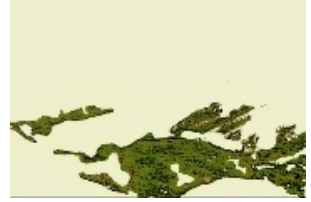

(d) For "grass": $K=3$, $|\mathbf{N}|=16, \zeta_{1}^{4.5 \%}=0.49$

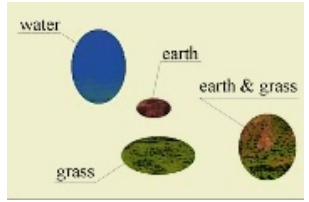

(b) Training samples

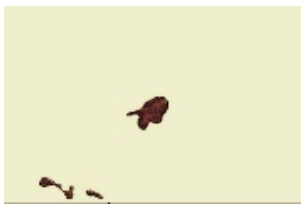

(e) For "earth": $K=2$, $|\mathbf{N}|=16, \zeta_{1}^{7.7 \%}=0.54$

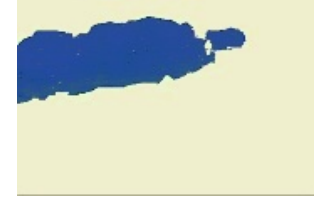

(c) For "water": $K=3$, $|\mathbf{N}|=11, \zeta_{2}^{9.6 \%}=0.92$

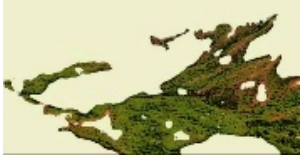

(f) For "earth \& grass": $K=3,|\mathbf{N}|=16, \zeta_{2}^{8 \%}=0.54$

Fig. 2. Results of detecting for different training samples using $\chi^{2}$-distance measure from the image ValleyWater.2.

parameters are chosen as follows: the moving window $W_{x} \times W_{y}=17 \times 17$, only one $(|\mathbf{A}|=1)$ most energetic family of pixel pairs per each training sample $\mathbf{G}^{t r}$ selected within all the relative shifts $\left(\xi_{a} \leq W_{x}, \eta_{a} \leq W_{y}\right)$, the maximum rejection rate $\hat{\rho}=20 \%$, the $\chi^{2}$-distance measure in Eq. (3) and $\bar{\zeta}=0.5$. The detecting results with the different training samples are illustrated in (c)-(f) in Fig. 1 and 2 respectively, the white areas are the rejected regions. Note that $K$ is the number of pyramid levels in Eq. (1), the subscript $L$ and superscript $\rho$ of $\zeta_{L}^{\rho}$ represents the stop level and the rejection rate, respectively. Clearly, the training areas can be always detected. The similar regions to the training area are almost found out. Meanwhile, most of these training regions are inhomogeneous, the results can be refined by using more levels of pyramids.

A special colour aerial image in (a) of Fig. 3 provided by the Institute of Communication Theory and Signal Processing (TNT), University of Hannover, is used below as a test example to search for each of the four training regions in (b) of Fig. 3 : field (homogeneous), vegetation (homogeneous), residential area (weakly homogeneous), and industrial area (inhomogeneous). The curves in (a) of Fig. 4 illustrate the empirical distributions of the quantized distances $D_{j}^{0}\left(\mathbf{F}_{j}\left(\mathbf{G}^{t r}\right), \mathbf{F}\left(\mathbf{G}^{t r}\right)\right)$ for their base level $L=0$ only (see Eq. (2)) over the training samples, using $\chi^{2}$-distance measure with $D_{\max }^{0}$ as the distance threshold (i.e. $\rho=0 \%$ ). The narrower the range of distances, the more homogeneous the texture. It is reasonable to set $\bar{\zeta}=0.5$ being the threshold of homogeneity for $\chi^{2}$-distance measure. In the same way, $\bar{\zeta}=0.5$ for fidelity measure and $\bar{\zeta}=0.25$ for quadratic form (QF) from the curves in (b) and (c) of Fig. 4, respectively.

Meanwhile the ratio between $\bar{\zeta}$ and a distance upbound $\hat{D}$ is $0.25,0.5$, and 0.176 for the $\chi^{2}$-distance, fidelity measure, and QF distance respectively, we can expect that the QF has the best performance and the $\chi^{2}$-distance can perform better than fidelity measure. This can be verified by the comparison using dif- 

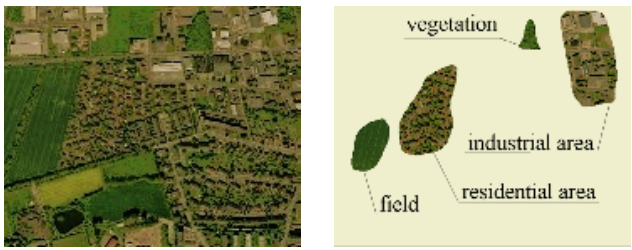

(a) Original aerial image (b) Training samples
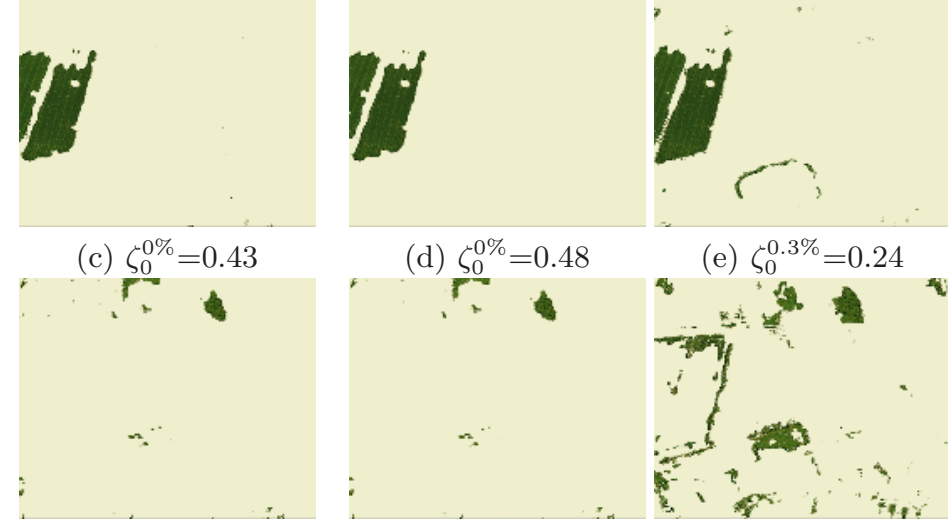

(e) $\zeta_{0}^{0.3 \%}=0.24$

(f) $\zeta_{0}^{0 \%}=0.24$

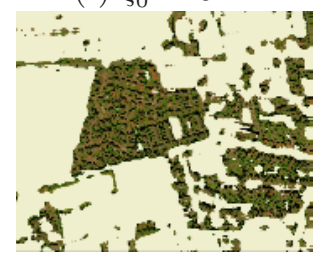

(g) $\zeta_{0}^{0 \%}=0.35$

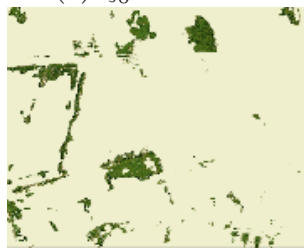

(i) $\zeta_{0}^{0.15 \%}=0.45$

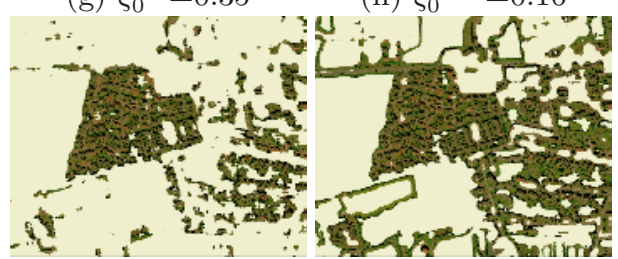

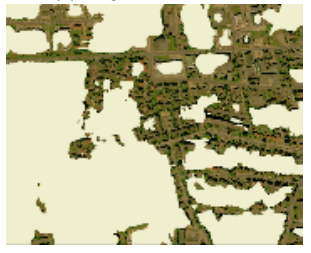

(l) $\zeta_{2}^{9.4 \%}=0.47$ $\chi^{2}$-distance (j) $\zeta_{0}^{1.2 \%}=0.47$

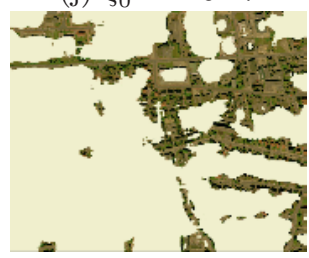

(m) $\zeta_{2}^{12.4 \%}=0.49$

fidelity measure (k) $\zeta_{0}^{1.6 \%}=0.21$

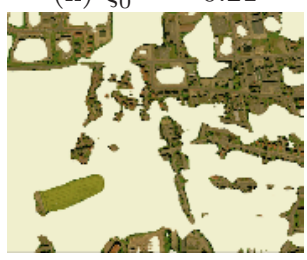

(n) $\zeta_{2}^{10.3 \%}=0.22$

QF distance

Fig. 3. Comparison using different distance measures: $\chi^{2}$-distance (left), fidelity measure (middle), and QF distance(right) with different training samples: "field" (second row), "vegetation" (third row), "residential area" (fourth row), and "industrial area" (fifth row), $|\mathbf{N}|=16$.

ferent distance measures with the different training samples demonstrated in (c)-(n) of Fig. 3. But QF is too time consuming so that it practically cannot be applied when $|\mathbf{N}|>16$. Furthermore, we can see that the performance for the homogeneous training samples "field" and "vegetation", and for weakly ho- 


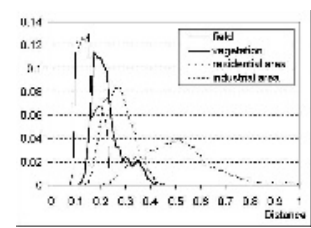

(a) $\chi^{2}$-distance

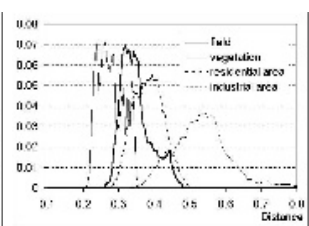

(b) fidelity measure

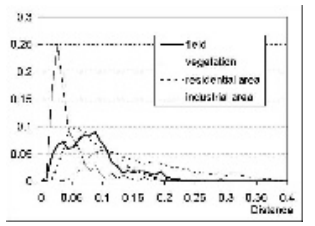

(c) quadratic form $(\mathrm{QF})$

$0 \leq d\left(\mathbf{f}_{a}, \mathbf{f}_{b}\right) \leq \sqrt{2}$

Fig. 4. Empirical distance distributions of different training samples in (b) of Fig. 3 using different distance measures. $W_{x} \times W_{y}=17 \times 17,|\mathbf{N}|=16,|\mathbf{A}|=1$.

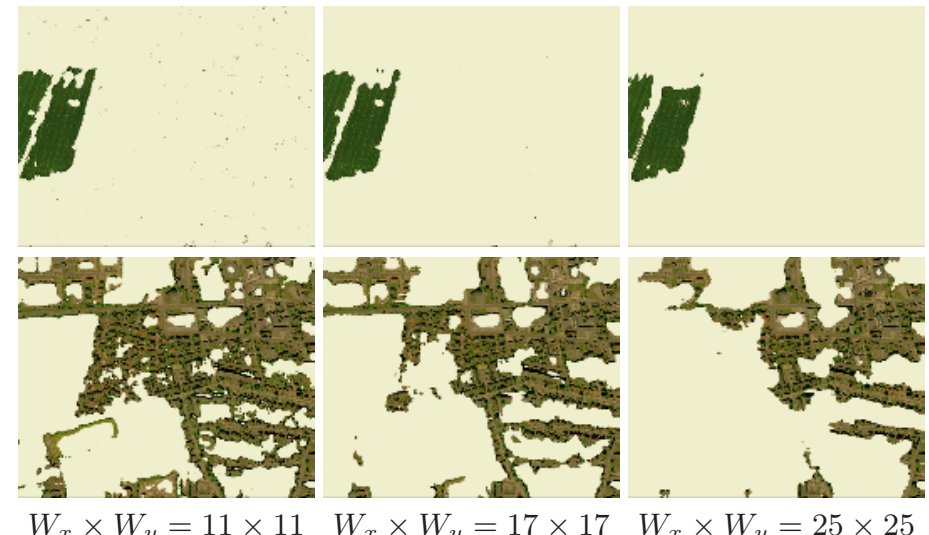

Fig. 5. Results with different size of moving window $W_{x} \times W_{y}$ for "field" (top row) and "industrial area" (bottom row) using $\chi^{2}$-distance measure $|\mathbf{N}|=16$.

mogeneous "residential area" are very good, except for a little border problem for "vegetation" because of its too small training area. For the inhomogeneous "industrial area", it still can get reasonably good performance, but some homogeneous sub-regions such as the large area roofs which exist in the training sample cannot be detected, the reason is that the $\mathrm{CCH}$ for the chosen clique family from the whole training sample has not reflect the one over these homogeneous sub-regions. Some roads are detected both for "residential area" and "industrial area" because similar road information exists in both training samples.

Our experiments show that more colours and large number of characteristic families cannot significantly improve the performance. Generally the suitable range of the colours number $N$ from the texture analysis viewpoint can be $16 \leq$ $N \leq 32$.

Figure 5 shows the detecting results with different size of moving window $W_{x} \times W_{y}$ using $\chi^{2}$-distance measure. For homogeneous textures such as "field", the results vary insignificantly with the different size of moving window within this range, and the border problem can be worse under the large size of moving window; however, the bigger size can get better performance for inhomogeneous textures such as "industrial area". 
In total, the proposed algorithm effectively detects a given colour texture on an arbitrary background.

\section{Acknowledgements}

This work was supported by the Royal Society of New Zealand Marsden Fund under Grant 3600771/9143 (UOA122).

\section{References}

1. S. K. Chang and A. Hsu. Image information systems: Where do we go from here? IEEE Trans. Knowledge and Data Engineering, 4(5):431-442, 1992.

2. D. De Ridder, D. M. J. Tax, and R. P. W. Duin. An experimental comparison of one-class classification methods. In Proc. of Advanced School for Computing and Imaging, Delft, June 1998.

3. G. L. Gimel'farb. Image Textures and Gibbs Random Fields. Kluwer Academic Publishers, Dordrecht, 1999.

4. J. Hafner, H. Sawhney, W. Equitz, M. Flickner, and W. Niblack. Efficient color histogram indexing for quadratic form distance functions. IEEE Trans. Pattern Analysis and Machine Intelligence, 17(7):729-736, 1995.

5. J. Lin. Divergence measures based on the shannon entropy. IEEE Trans. Information Theory, 37(1):145-151, 1991.

6. M. M. Moya and D. R. Hush. Network constraints and multi-objective optimization for one-class classification. Neural Networks, 9(3):463-474, 1996.

7. M. Nolle. Distribution distance measures applied to 3-d object recognition - a case study. In Proc. the 25th Pattern Recognition Symposiom of the German Association for Pattern Recognition, Magdeburg, Germany, Sept. 2003.

8. J. Puzicha, Y. Rubner, C. Tomasi, and J. Buhmann. Empirical evaluation of dissimilarity measures for color and texture. In Proc. IEEE Int. Conf. on Computer Vision (ICCV-1999), pages 1165-1173, 1999.

9. Y. Rui, T. S. Huang, and S. Chang. Image retrieval: current techniques, promising directions and open issues. J. Visual Communication and Image Representation, 10(4):39-62, 1999.

10. A. W. M. Smeulders, M. Worring, S. Santini, A. Gupta, and R. Jain. Content based image retrieval at the end of the early years. IEEE Trans. Pattern Analysis and Machine Intelligence, 22(12):1349-1380, 2000.

11. R. E. Sánchez-Yáñez, E. V. Kurmyshev, and F. J. Cuevas. A framework for texture classification using the coordinated clusters representation. Pattern Recognition Letters, 24:21-31, 2003.

12. L. Yu and G. Gimel'farb. Pairwise pixel interactions in texture pyramids. In Image and Vision Computing New Zealand, pages 209-214, Auckland, New Zealand, November 2002.

13. L. Yu and G. Gimel'farb. Image retrieval using colour co-occurrence histograms. In Image and Vision Computing New Zealand, pages 42-47, Palmerston North, New Zealand, November 2003.

14. L. Yu and G. Gimel'farb. Separating a colour texture from an arbitrary background. In 7th Biennial Australian Pattern Recognition Society Conference on Digital Image Computing: Techniques and Applications, volume 1, pages 489-498, Sydney, Australia, December 2003. 